Harvard Kennedy School Misinformation Review ${ }^{1}$

June 2020, Volume 1, Special Issue on COVID-19 and Misinformation

Creative Commons Attribution 4.0 International (CC BY 4.0)

Reprints and permissions: misinforeview@hks.harvard.edu

DOI: https://doi.org/10.37016/mr-2020-027

Website: misinforeview.hks.harvard.edu

\title{
Promoting health literacy during the COVID-19 pandemic: $A$ call to action for healthcare professionals
}

The extraordinary spread of misinformation during the COVID-19 pandemic is impressive. And, to public health professionals like us, it's worrying: We know that good information and good health go hand in hand. Knowing what we do about the practice of public health and what the science tells us about how people fall for misinformation, we see promising strategies for intervention in our own field. We therefore call on fellow healthcare professionals to take concerted action against misinformation, and we suggest here one lever our field is perfectly situated to address: health literacy. In this commentary, we propose concrete strategies for colleagues at four levels of practice: in healthcare organizations, community-based partnerships, cross-sector collaborations, and as individual healthcare providers.

Authors: April Joy Damian (1), Joseph J. Gallo (1)

Affiliations: (1) John Hopkins Bloomberg School of Public Health, USA

How to cite: Damian, A. J., \& Gallo, J. J. (2020). Promoting health literacy during the COVID-19 pandemic: A call to action for healthcare professionals. Harvard Kennedy School (HKS) Misinformation Review, 1(3).

Received: March 26 $6^{\text {th }}, 2020$. Accepted: May 22 ${ }^{\text {nd }}, 2020$. Published: June $15^{\text {th }}, 2020$.

\section{Introduction}

Health literacy is crucial for health, and healthcare professionals must acknowledge and prioritize its importance in every interaction with patients and communities (Berkman et al., 2011; Paasche-Orlow \& Wolf, 2007). But we can't stop there. Given its impact on health itself, health literacy merits a privileged position in our entire scope of operations, from program planning to evaluation to patient safety to communications. A strong body of evidence testifies to the benefits of integrating individual healthcare practice with public health strategy, and the current pandemic is the perfect moment for professionals from these two sectors to join forces in a shared battle against misinformation (Linde-Feucht \& Couluris, 2012; Committee on Integrating Primary and Public Health, Board on Population Health and Public Health Practice \& Institute of Medicine, 2012). In the midst of what the WHO has called a "misinfodemic," the stakes of health literacy - or illiteracy - are high, and we consider it our duty, as healthcare professionals, to do everything we can to help people access and act on the sort of health-related information that effectively promotes the achievement of good health. A strengthened commitment to health literacy throughout the entire family of healthcare professionals, within and across organizations and down to each individual provider, offers us great promise for addressing this urgent problem. Here we show why and how.

\footnotetext{
${ }^{1}$ A publication of the Shorenstein Center for Media, Politics and Public Policy, at Harvard University, John F. Kennedy School of Government.
} 


\section{Background}

Before officially declaring COVID-19 a pandemic, the World Health Organization described the massive infodemic surrounding it, pointing to a global overabundance of information that had already made it difficult for the public to distinguish trustworthy health sources from phony experts and created widespread confusion about real evidence-based safety measures (Zarocostas, 2020). Indeed, a recent Pew survey of American adults found that close to half (48\%) of the respondents reported seeing at least some made-up news about COVID-19 (Mitchell \& Oliphant, 2020).

Research suggests that several key factors drive individual and collective belief in health-related misinformation (Scheufele \& Krause, 2019; Uscinski et al., 2016). One of the most important is low health literacy - and the extraordinarily scope and scale of the current COVID-19 "misinfodemic" represents an acute challenge to healthcare professionals in just this regard (Gyenes \& Marrelli, 2019; Mian \& Khan, 2020). History has shown us the tragic consequences that the spread of misinformation can carry during health crises. (For example, the South African government's outright denial of the efficacy of antiretroviral therapies and its mass promotion of herbal remedies to combat HIV/AIDS under President Thabo led to the loss of over 300,000 lives as a result of excess HIV transmission, particularly among pregnant mothers)(Nlooto \& Naidoo, 2016; Bateman, 2007). The Affordable Care Act (2010) defines health literacy as "the degree to which an individual has the capacity to obtain, communicate, process, and understand health information and services to make appropriate health decisions." In short, it is the sort of competence that enables people to recognize the severity of a given situation, acquire a basic grasp of how to protect themselves and others, and understand the scope of their choices. In the context of COVID19 , it is what makes people able to differentiate between proposals to take social distancing measures and ones to drink bleach.

While limited health literacy affects people of all ages, incomes, and education levels, some are more vulnerable than others. According to the Centers for Disease Control and Prevention (CDC), racial and ethnic minorities, non-native speakers of English, those with low socioeconomic status, and medically underserved people (e.g., the uninsured and Medicaid-insured) are disproportionately susceptible. Hispanic adults have the lowest average health literacy scores of all racial and ethnic groups, followed by African Americans and American Indian/Alaska Natives; one study found that $74 \%$ of Spanish-speaking patients, for example, demonstrated "below basic" health literacy (the lowest level possible), while only $7 \%$ of English-speaking patients did. And disadvantages such as these matter because there are serious consequences: People with low health literacy are twice as likely as others to report poor health status (Cutilli \& Bennett, 2009).

Recent scientific work has affirmed that misinformation (generally) and limited health literacy (specifically) are major obstacles to health, and have helped to explain and respond to the problem (The National Academies of Sciences, Engineering, and Medicine, 2016; The National Academies of Sciences, Engineering, and Medicine, 2017). In 2017, the National Academies of Sciences (NAS) convened a Committee on the Science of Science Communication to highlight the challenges inherent in effectively conveying scientific information to the public. Additional thought leaders in the field have built on the committee's work by recognizing misinformation as a product of individual information processing as well as of macro-level causes that include the pervasiveness of social networks and the tenure of highly varied information ecologies (Scheufele \& Krause, 2019). Previous studies have noted a lack of understanding of science, the inability to recognize misinformation, the holding of beliefs inconsistent with the best available science, and credence in conspiracy theories as a few of the individual factors associated with the acceptance of inaccurate information (Uscinski et al., 2016; Garrett \& Weeks, 2017; Southwell \& Thorson, 2015). Other research has suggested that emotional states can influence individuals' ideologies 
and alter their propensity to assimilate falsehoods from the information environment (Kalichman et al., 2012; Flynn, Nyhan \& Reifler, 2017).

Group-level dynamics have also been found to influence individuals' uptake of misinformation. For example, insular social networks can shape people's attitudes and beliefs, allowing for the normalization of falsehoods and making it difficult to counter unfounded beliefs with correct information (DiFonzo et al., 2013). There are also major systems-level factors that contribute to the spread of misinformation in the United States, including: (1) declining social capital; (2) growing economic inequalities; (3) increasing political polarization; (4) declining trust in science; (5) politically asymmetric credulity; (6) an evolving media landscape; and (7) a fractioning of the media that rewards political extremism (The National Academies of Sciences, Engineering, and Medicine, 2017).

Understanding how these individual-, group-, and systems-level factors condition health literacy gives us clues about how misinfodemics are born and spread. But they also suggest measures that might be levered to develop intervention strategies and solutions to this slippery problem. We have identified a few such strategies, and we issue to healthcare professionals of all sorts a call to action. In the following recommendations, we propose health literacy promotion approaches that can be taken by four key professional stakeholder groups: healthcare organizations; community-based partnerships; cross-sector collaborations; and individual providers.

\section{Healthcare organizations should strengthen their commitment to health literacy across the scope of operations}

Healthcare systems are staffed by a clinical workforce that can leverage its therapeutic relationships with patients to promote and track progress in health literacy. Organizational leadership should avail of these relationships to prioritize the promotion of health literacy in all its outward-facing interactions, integrating health literacy content into planning, evaluation, communication, and patient safety strategies (Brach, 2017). It should identify appropriate "teachable moments" for addressing misinformation - such as care transitions, the prescription of medicines, and vaccination appointments - and plan appropriate health literacy interventions for each one.

Community health centers are sites of great potential for the promotion of health literacy. For over 50 years, health centers funded by the U.S. Health Resources and Services Administration (HRSA) have functioned as the nation's primary care backbone for the medically underserved, coordinating care for over 29 million people across the U.S. And since part of these health centers' statutory mission is to provide comprehensive healthcare to the medically underserved regardless of their insurance status or ability to pay, their patients include 1 of every 3 people living in poverty, 1 in 5 rural residents, 1 in 5 uninsured persons, 1 in 6 Medicaid beneficiaries, 1.4 million homeless people, and 385,000 veterans. Its more than 12,000 service delivery locations reach every state and territory, and $45 \%$ are in rural areas (National Association of Community Health Centers, 2019). If the healthcare organizations that manage these health centers recognize their potential, they could become key sites for promoting health literacy among the most vulnerable members of society - and for combatting the misinfodemics that thrive in just those contexts of exclusion where health literacy does not. 


\section{Healthcare professionals should build and enhance partnerships with the communities they serve}

Healthcare organizations and providers already interact with the populations they serve, but strengthening relationships with diverse community organizations, congregations, and non-medical service agencies will strengthen their position in communities and open new opportunities for information exchange. For example, community-targeted forums can be organized in locally important venues such as churches, recreation centers, and community centers and designed to deliver and discuss relevant health information with the public. Similarly, local organizations can be recruited to serve as direct sources of timely, accurate information, leveraging their existing relationships and rapport with individuals and communities. These entities have an insider's perspective and can offer valuable insights regarding the best ways to disseminate information and help to reach even the hardest-to-reach members of their communities.

Establishing strong partnerships with local organizations is especially important since the oral cultures of many communities make personal interactions crucial for information dissemination. At the same time, there is a strong scientific evidence base supporting participatory program design processes and demonstrating the benefits of involving members of target audiences in the design and testing of communication products (Cyril et al., 2015).

\section{Healthcare professionals should reinforce their cross-sector collaborations with public health systems}

In 2010, The Affordable Care Act expanded health insurance coverage for millions of Americans. But it also highlighted the need for healthcare and public health systems to work together to address individual health within the context of the larger community, incentivizing practices such as accountable care organizations and patient-centered medical homes that adopt integrative strategies (42 U.S.C. § 18001). Two years later, recognizing similar benefits, the Institute of Medicine's (IOM) Committee on Integrating Primary Care and Public Health outlined a set of recommendations for aligning primary care with public health strategy. Its review examined over 400 collaborations between medical and public health actions and identified benefits for both primary care providers (such as the provision of population-based information that effectively enhanced their capacity to address behavioral and other underlying causes of illness) and public health entities (which received support from primary care providers in the execution of population-based strategies such as the collection of individual-level data for surveillance purposes and the dissemination of health education and health promotion messages) (Committee on Integrating Primary and Public Health, Board on Population Health and Public Health Practice \& Institute of Medicine, 2012; Lassker, 1997).

Similarly, just last year, the American Academy of Family Physicians (AAFP) issued a position paper insisting on the importance and value of primary care and public health integration and urging its members to pursue and support integrative collaborations and interventions (American Academy of Family Physicians, 2015).

The current crisis is a prime opportunity for healthcare and public health professionals to build on their growing legacy of collaboration to partner once more, joining forces now to combat the misinfodemic that threatens the health and well-being of individuals and populations alike. 


\section{Individual healthcare providers should make every effort to understand and respond to their patients' unique health-related knowledge, belief, and literacy conditions}

A 2018 Gallup poll found that four in five Americans (84\%) rated the honesty and ethical standards of nurses as "very high" or "high," making them the most well-regarded professionals among those considered; doctors ranked second at $67 \%$ (Brenan, 2018). Another Gallup poll, conducted this March in the midst of the COVID-19 pandemic, produced similar findings, showing hospitals and health agencies to be the most trustworthy of the nine major national institutions and leaders proposed (Newport, 2020). Despite this privileged social regard, however, many front-line providers remain unaware of their patients' beliefs - because they never ask. The notion of a personal explanatory model of illness refers to a patient's representation of disease and is based on beliefs, emotions, knowledge, and experiences that are grounded in culture (Kleinman, 1980).

These representations of disease are associated with illness-related beliefs (such as how strongly they believe in self-control over health) and behaviors (such as how long patients stay in treatment) (Lau et al., 1989; Leventhal et al., 1980). While these personal explanatory models can differ radically from scientific models concerning the causation of disease, what people actually do in the face of illness is often a logical extension of their personal beliefs rather than of the scientific standard (Good, 1992). Individual doctors must recognize, then, that understanding a patient's beliefs is the starting point for engaging in a discussion of how the patient encounters her world and what matters most to her - and that can become an important, personal, and personalized opportunity to correct misinformation, to improve health literacy, and - ultimately - to improve health.

\section{Reiterating our call to action: A multi-level healthcare response to the misinfodemic}

Countering the COVID-19 misinfodemic is a critical task for individual and public health. Healthcare professionals in all sectors and at all levels must strengthen their commitment to health literacy. Here we have issued four recommendations describing how healthcare organizations, community-based partnerships, cross-sector collaborations, and individual healthcare providers can play roles in the fight against misinformation by strengthening their efforts to build health literacy among the people and populations they serve.

And play a role they must: This battle is scored in lives and deaths.

\section{Bibliography}

Affordable Care Act of 2010, 42 U.S.C. § 18001 et seq. (2010). https://www.law.cornell.edu/uscode/text/42/18001

American Academy of Family Physicians. (2015). Integration of primary care and public health (position paper). https://www.aafp.org/about/policies/all/integration-primary-care.html

Berkman, N. D., Sheridan, S. L., Donahue, K. E., Halpern, D. J., \& Crotty, K. (2011). Low health literacy and health outcomes: An updated systematic review. Annals of Internal Medicine, 155(2), 97-107. https://doi.org/10.7326/0003-4819-155-2-201107190-00005

Brach, C. (2017). The journey to become a health literate organization: A snapshot of health system improvement. Studies in Health Technology and Informatics, 240, 203-237. 
Brenan, M. (2018, December 20). Nurses again outpace other professions for honesty, ethics. Gallup. https://news.gallup.com/poll/245597/nurses-again-outpace-professions-honesty-ethics.aspx

Committee on Integrating Primary and Public Health, Board on Population Health and Public Health Practice \& Institute of Medicine. (2012). Primary care and public health: Exploring integration to improve population health. The National Academies Press. https://doi.org/10.17226/13381

Cutilli, C. C., \& Bennett, I. M. (2009). Understanding the health literacy of America: Results of the national assessment of adult literacy. Orthopaedic Nursing, 28(1), 27-32. https://doi.org/10.1097/01.NOR.0000345852.22122.d6

Cyril, S., Smith, B. J., Possamai-Inesedy, A., \& Renzaho, A. M. N. (2015). Exploring the role of community engagement in improving the health of disadvantaged populations: A systematic review. Global Health Action, 8(1), 1-12. https://doi.org/10.3402/gha.v8.29842

DiFonzo, N., Bourgeois, M. J., Suls, J., Homan, C., Stupak, N., Brooks, B. P., Ross, D. S., \& Bordia, P. (2013). Rumor clustering, consensus, and polarization: Dynamic social impact and selforganization of heresay. Journal of Experimental Social Psychology, 49(3), 378-399. https://doi.org/10.1016/j.jesp.2012.12.010

Flynn, D. J., Nyhan, B., \& Reifler, J. (2017). The nature and origins of misperceptions: Understanding false and unsupported beliefs about politics. Political Psychology, 38(S1), 127-150.

Garrett, R. K., \& Weeks, B. E. (2017). Epistemic beliefs' role in promoting misperceptions and conspiracist ideation. PLoS One, 12(9), e0184733. https://doi.org/10.1371/journal.pone.0184733

Good, B. J. (1992). Culture and psychopathology: Direction for psychiatric anthropology. In T. Schwartz, G. M. White \& C. A. Lutz (Eds.), New Directions in Psychological Anthropology (pp. 181-205). Cambridge University Press.

Gyenes, N., \& Marrelli, M. (2019). Health equity through health fact-checking: A primer. Meedan Digital Health Lab. https://health.meedan.com/primer.pdf

Kalichman, S. C., Cherry, C., \& White, D. (2012). Use of dietary supplements among people living with HIV/AIDS is associated with vulnerability to medical misinformation on the internet. AIDS Research and Therapy, 9(1), 1. https://doi.org/10.1186/1742-6405-9-1

Kleinman, A. (1980). Patient and healer in the context of culture: An exploration of the borderland between anthropology, medicine, and psychiatry. University of California Press.

Lassker, R. D. (1997). Medicine and public health: The power of collaboration. Health Administration Press.

Lau, R. R., Bernard, T. M., \& Hartman, D. T. (1989). Further explorations of common-sense representations of common illness. Health Psychology, 8(2), 195-219. https://doi.org/10.1037/0278-6133.8.2.195

Leventhal, H., Meyer, D., \& Nerenz, D. T. (1980). The common sense representation of illness danger. In S. Rachman (Ed.), Contributions to Medical Psychology (pp. 7-30). Pergamon Press.

Linde-Feucht, S., \& Coulouris, N. (2012). Integrating primary care and public health: A strategic priority. American Journal of Public Health, 102(3), S310-311. https://doi.org/10.2105/AJPH.2012.300849

Mitchell, A., \& Oliphant, J. B. (2020, March 18). Americans immersed in COVID-19 news; most think media are doing fairly well covering it. Pew Research Center. https://www.journalism.org/2020/03/18/americans-immersed-in-covid-19-news-most-thinkmedia-are-doing-fairly-well-covering-it/

National Association of Community Health Centers. (2019). America's health centers. http://www.nachc.org/wp-content/uploads/2019/09/Americas-Health-Centers-Updated-Sept2019.pdf 
Newport, F. (2020, March 27). Six emerging conclusions: Public opinion and COVID-19. Gallup. https://news.gallup.com/opinion/polling-matters/304646/five-emerging-conclusions-publicopinion-covid.aspx

Passche-Orlow, M. K., \& Wolf, M. S. (2007). The causal pathways linking health literacy to health outcome. American Journal of Health Behavior, 31(1), 19-26.

Scheufele, D. A., \& Krause, N. M. (2019). Science audiences, misinformation, and fake news. PNAS, 116(16), 7662-7669. https://doi.org/10.1073/pnas.1805871115

Southwell, B. G., \& Thorson, E. A. (2015). The prevalence, consequence, and remedy of misinformation in mass media systems. Journal of Communication, 65(4), 589-595. https://doi.org/10.1111/jcom.12168

The National Academies of Sciences, Engineering, and Medicine. (2016). Science literacy: Concepts, contexts, and consequences. The National Academies Press. https://doi.org/10.17226/23595

The National Academies of Sciences, Engineering, and Medicine. (2017). Communicating science effectively: A research agenda. The National Academies Press. https://doi.org/10.17226/23674

Uscinski, J. E., Klofstad, C., Atkinson, M. D. (2016). What drives conspiratorial beliefs? The role of information cues and predisposition. Political Research Quarterly, 69(1), 57-71. https://doi.org/10.1177/1065912915621621

Zatocostas, J. (2020). How to fight an infodemic. The Lancet, 395(10225), 676. https://doi.org/10.1016/S0140-6736(20)30461-X 


\section{Copyright}

This is an open access article distributed under the terms of the Creative Commons Attribution License, which permits unrestricted use, distribution, and reproduction in any medium, provided that the original author and source are properly credited. 